\section{Remote Microscopy for Education and Outreach}

Supapan Seraphin, Steven Hernandez, Gary Chandler, David Bentley ${ }^{\star}$, Kristina Dorame, Marilee Sellers** Department of Materials Science and Engineering, University of Arizona, Tucson, AZ

* Arizona Research Laboratory, University of Arizona, Tucson, AZ

** Imaging and Histology Core Facility, Northern Arizona University, Flagstaff, $A Z$

Scanning electron microscopy and X-ray analysis are standard tools in the nanotechnology world, but access to them by students is limited by high cost and lack of equipment. For this reason, among others, we have developed an on-demand remote access system to The University Spectroscopy and Imaging Facilities (USIF) [1] of The University of Arizona (UA), by using Quartz PCI-Taipan [2]. In general, Taipan is an online server based program that enables spectroscopy laboratories to store, organize, and manage data as well as users. Significantly, the Taipan system allows users, operators and researches to create their own online collaborations, which will, in many ways, fuse their research and outreach efforts. Therefore, we have incorporated the Taipan software to our recently installed Hitachi S-4800 FESEM and S-3400N variable-pressure SEM. With a high-speed internet connection, from any remote setting, the system allows students real-time access to view state-of-the-art microscopes, acquire data, and interact with the instrument operator. What is more, remote users can view up to six different screens including SEM image, SEM control screen, EDS, Raman spectrum, chamber scope, and microscope room as shown in Fig. 1. This offers a significant reduction of time, travel expenses, and more importantly, equipment cost.

One of our main challenges, when integrating the software with the lab, was the security used in the UA computer network. Yet, after much work, we were successful in connecting with other computers via the Taipan collaboration interface. One success, was initiating a collaboration session with the Imaging and Histology

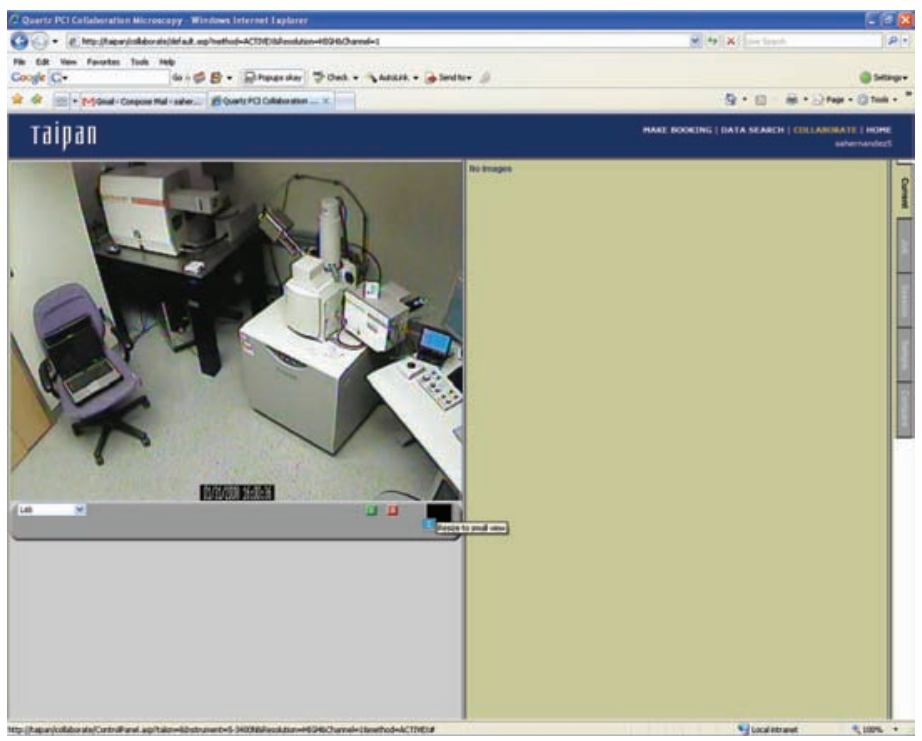

Fig. 1 Image of $S 3400-N$ in real time from a camera. A remote user would have a similar view of the microscopes and users in our laboratory.
Core Facility at Northern Arizona University (NAU). The Taipan collaborative software has allowed NAU faculty to reserve instrument time and to view live operation on the instruments. This access system is portable to any classroom location with wired or wireless internet connections. The computer screen is projected by LCD projector onto a large classroom screen and can be shared with any size class. Fig. 2 shows file sharing interface on Taipan that allows users to access saved data at a remote location.

In spring 2008, Taipan collaboration sessions were conducted in classes such as Chemistry 462, Biochemistry II, at NAU and MSE 480, Experimental Methods for Microstructural Analysis, at UA. In addition, we held a successful Taipan collaboration session at The University of Arizona, USIF's Open House on March 19, 2008. In the Marley lecture hall, attendees were given a brief demonstration of Taipan's capabilities. Moreover, the open house included prominent speakers and demonstrations of the instruments and software.

Finally, a big step in testing the remote access system was at the workshop "Remote Operation Across the Pacific of Scanning Electron Microscopy and Optical Raman Spectroscopy Performed Simultaneously," held at the National Materials Technology Center, Bangkok, Thailand on June 11-13, 2008. Over one-hundred participants attended the lecture part of the workshop. Impressively, through Taipan, and along with Skype ${ }^{\mathrm{mx}}$ for voice communication, we were able to provide two successful remote sessions in two consecutive days during the workshop.
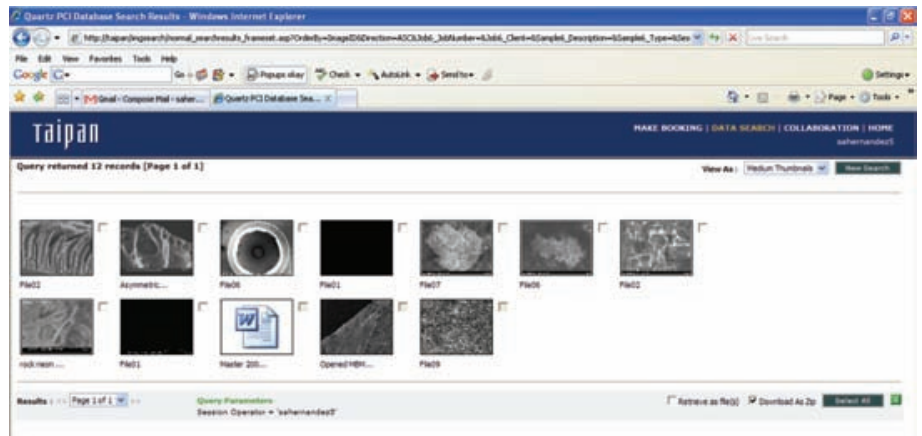

Fig. 2 File sharing interface on Taipan. This allows users to access or share saved data at a remote location.

Our major problems and solutions are listed below: Problem 1: inability of off-site users to download the necessary plug-ins on their computers.

Solution: Some users were unable to install the plug-ins without IT assistance. It is important for Taipan users to know what changes they can make to their computers, notably in a work or university environment.

Problem 2: Off-site users were unable to login to the Taipan site for collaboration sessions. 


\section{IIGMA}

\section{The Analytical FESEM Centered on EEMINI ${ }^{\circledR}$ Technology}

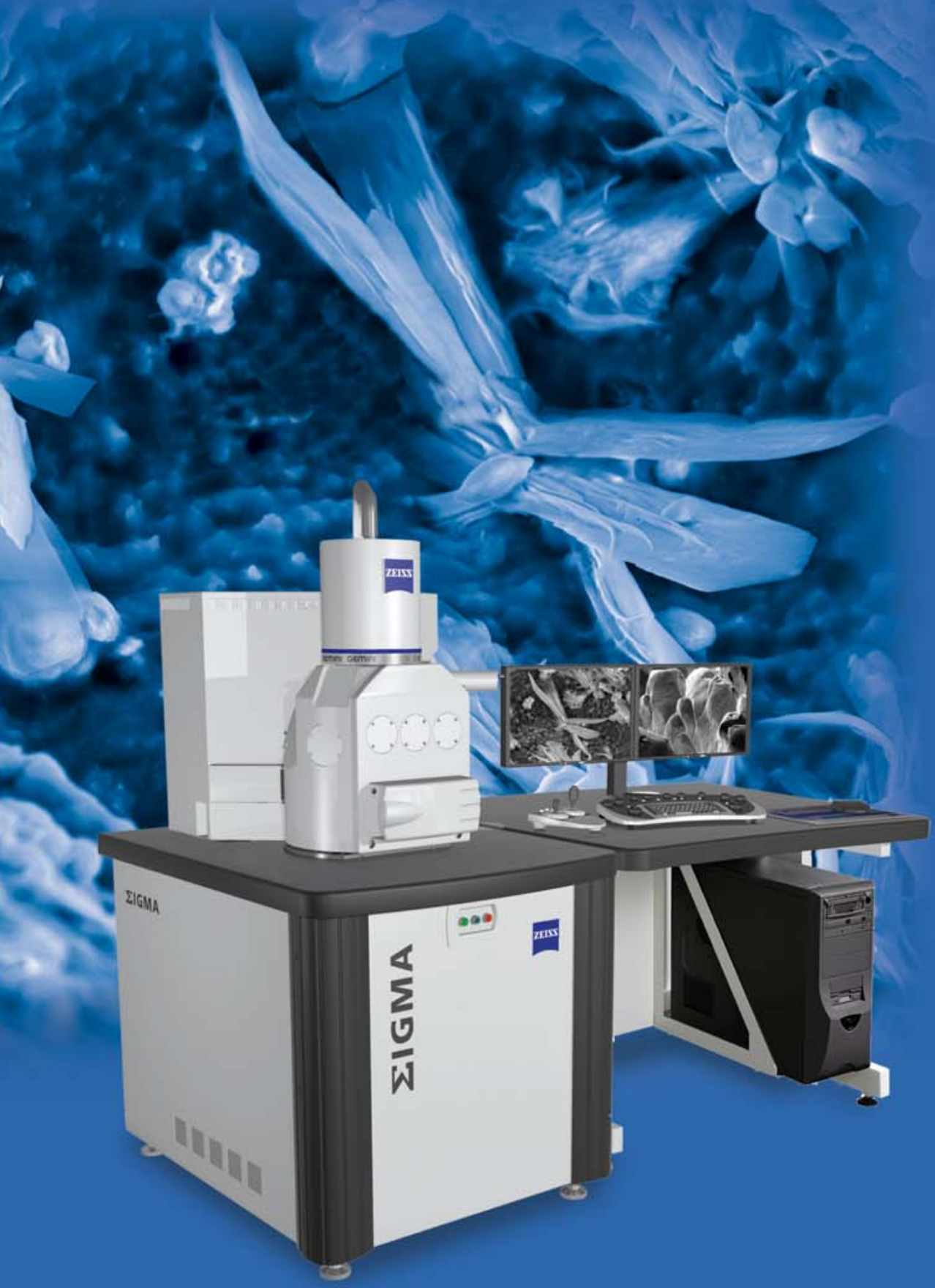

The $\Sigma$ IGMA, featuring GEMINI ${ }^{\circ}$ technology provides outstanding imaging and analytical results from a field emission microscope with the capability to handle all material types.

GEMINI is established as the market leading Field Emission design offering unrivalled ease of use, superb low voltage imaging and ultra stable probe currents for analytical applications.

Materials analysis at high resolution is provided by the class leading $X$-ray geometry for both energy (EDS) and wavelength dispersive spectroscopy (WDS).

The $\Sigma$ IGMA can handle specimens of up to $250 \mathrm{~mm}$ diameter and $145 \mathrm{~mm}$ tall. Furthermore, the coplanar chamber design provides the ideal geometry for simultaneous EDS and electron backscattered diffraction (EBSD).

Whatever the imaging challenges the IIGMA will make it visible.

- Peerless ease of use

- Unsurpassed imaging at low beam energy - Class leading X-ray and analytical geometry

\section{Carl Zeiss SMT Inc.}

Enabling the Nano-Age World ${ }^{*}$
One Corporation Way Peabody MA 01960 USA
Tel. $+1978 / 8267909$ Fax $+1978 / 5325696$ info-usa@smt.zeiss.com www.smt.zeiss.com/nts
ZDINX

We make it visible. 

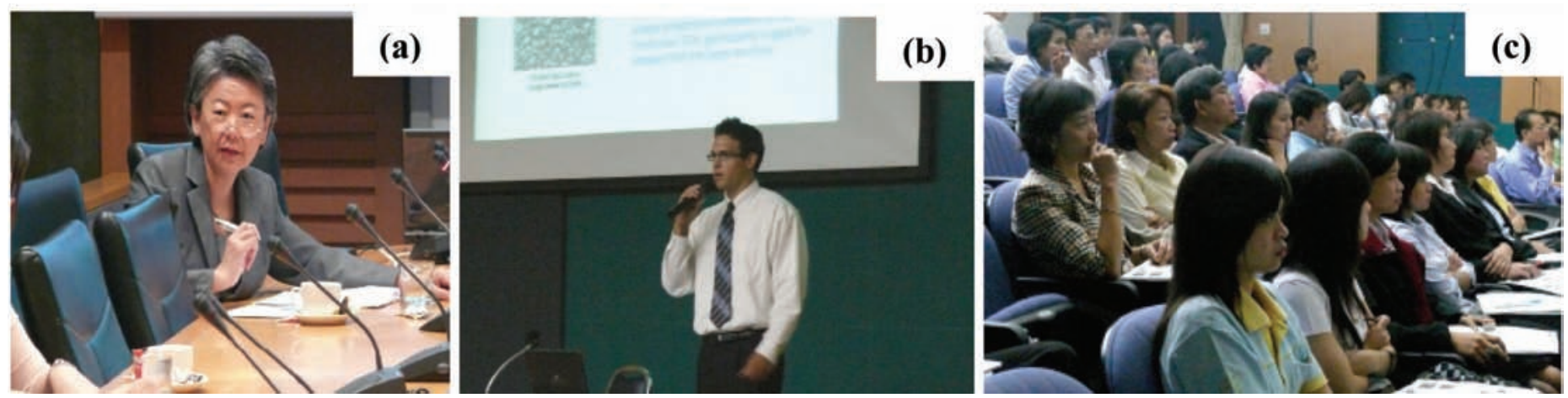

Fig. 3 Real-time remote-access collaboration session at SEM workshop in Thailand, June 11-13, 2008.

Solution: Our network administrators needed to open network ports to allow collaborators access to our system. Ports are names given to various network connections a computer uses to communicate with other computers. While this solution allowed remote access, it also made our network more susceptible to hackers, viruses, or worms; a more secure, permanent solution is a future goal.

\section{Problem 3: Use of cordless telephones or mobile phones made real-time communication difficult and unreliable.}

Solution: We installed USB computer microphones and speakers for use with Skype $^{\mathrm{Tm}}$ a free internet service that allows users to make voice calls from their computer to any other computer that has the Skype ${ }^{\mathrm{Tx}}$ program installed. This communication system is easy to use, provides clear sound, and allows for hands-free discussion, which is especially important for microscope operators and educators in the classroom. In addition, it is important to ensure that the Windows XP sound settings are optimized for use in conjunction with Skype.

\section{Recommendations for running Taipan Collaborations}

The following recommendations were developed as a result of countless hours spent developing and performing demos with the Taipan system. These recommendations offer a timeline of sorts to help those who are new to the system and to give them an idea of the preparation necessary to hold a seamless collaboration session.

\section{A. Month(s) prior to collaboration session}

(1) Remote collaborator and USIF SEM operator agree on samples to be used.

(2) Collaborators discuss audience and session plan.

(3) Appropriate microscope is selected and time is reserved.

(4) Resolve billing questions; get a purchase order number if necessary.

(5) Add remote collaboration to the Quartz-Taipan user database.

(6) Do a preliminary system test to verify our lab can be accessed remotely.

(7) Samples should be sent to USIF.

B. Week(s) prior to collaboration session

(1) USIF microscope operator should familiarize himself/ herself with the samples by evaluating them on a microscope.

(2) The remote collaborator and the USIF operator retest the system. This time the sample(s) to be used during the collaboration should be used. A concrete lesson plan should be agreed upon (if collaboration is to be done in a classroom).

\section{Day of collaboration}

(1) Microscope operators should give themselves 1-2 hours before collaboration session to setup equipment. This includes preparing for EDS or Renishaw SCA use if needed.

(2) If possible, they should also connect with the collaborator during this period when the internet connection is established.

(3) Once sample is in the microscope, the operator can align the column and find an interesting area of the sample.

(4) Check Skype connection. Make sure that the microphone is connected to a USB port.

An example of a lesson plan for MSE488/588 Scanning electron microscopy is:

\section{MSE 488/588 Taipan Collaboration}

Session Two: Standard Operation Procedures (week 2 in lecture) (All sessions are planned for 30 minute demonstrations to the class.)

1. Introduction of Microscope (Hitachi S-3400), staff.

2. Time-saving tips.

(assume high-resolution mode) (W thin film sample).

Low magnification: ability to find sample area.

Inclusion of "standard" on all samples (sample as THE

variable in analysis).

Column alignment.

Focus and stigmate: High Magnification.

Rapid working technique for beam-sensitive, charging, or contamination.

3. Image Modes: Choice of operating conditions.

Change CL strength.

Change WD.

4. Conclusions, summary (end Taipan session).

5. The SEM session as an experiment.

Sample or Microscope?

Operator's responsibility: Brightness equation.

Why study electron optics?

We continue to test the system and develop procedures that will work with various computer networks. One of the goals is to test collaboration curriculum for use in the classroom.

\section{References:}

[1] University of Arizona Spectroscopy and Imaging Facilities, http://imaging. arl.arizona.edu/

[2] Quartz PCI Taipan brochure, http://www.marinereef.com/pdfs/taipan. pdf 


\section{SII

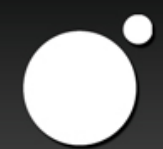

SII NanoTechnology USA Inc.

\section{Silicon Drift Detector}

NO LN2 • Active area $50 \mathrm{~mm}^{2} \bullet<130 \mathrm{eV}$ FWHM at $5.9 \mathrm{keV} \bullet$ ICR 1.5 Mcps $\bullet$ OCR up to $600 \mathrm{kcps}$

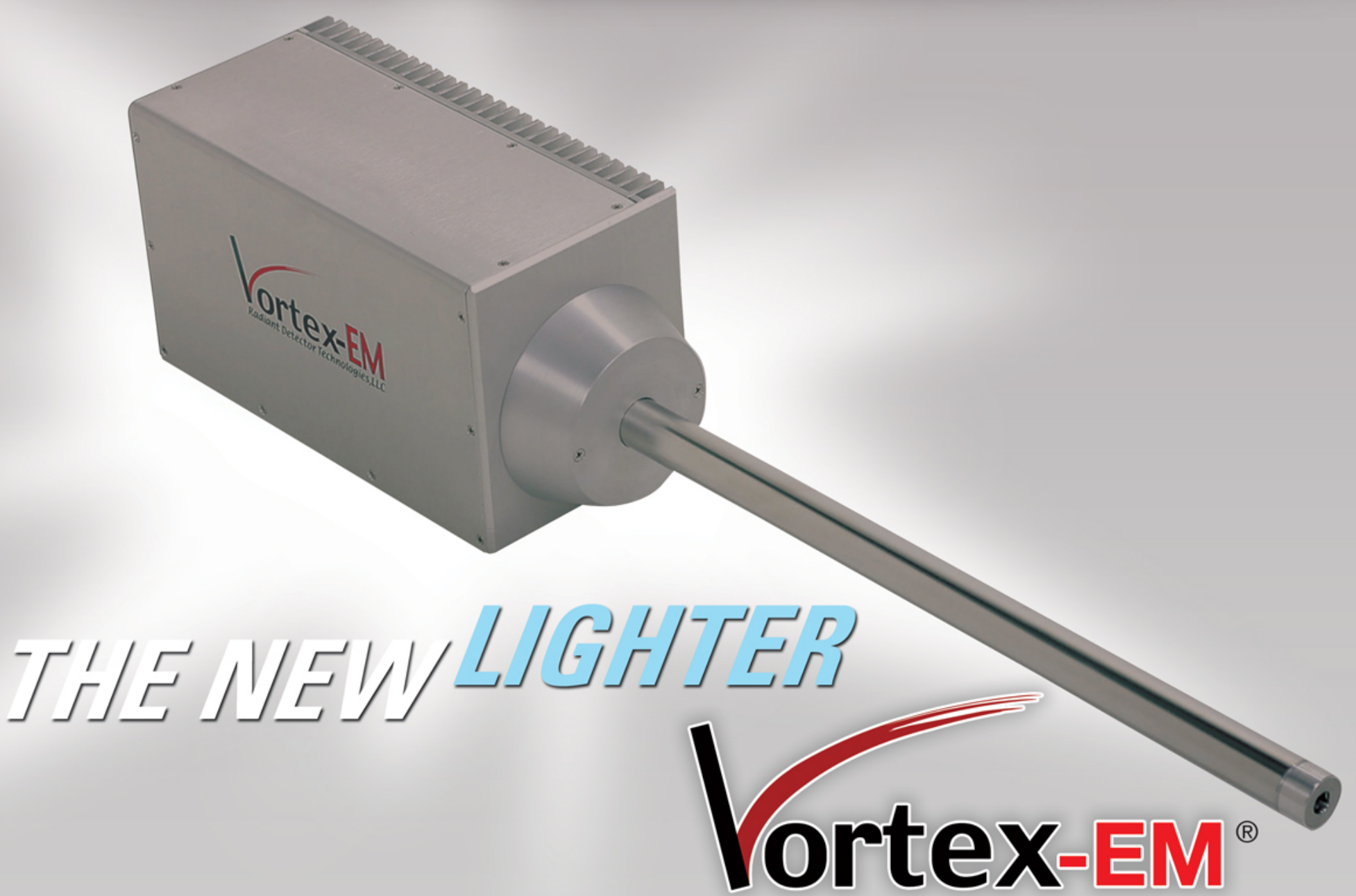

\section{BREAKTHROUGH PERFORMANCE}

C and the B peaks are completely separated from noise by the Vortex-EM detector. It therefore, facilitates light element detection while performing microanalysis and fast elemental mapping applications.

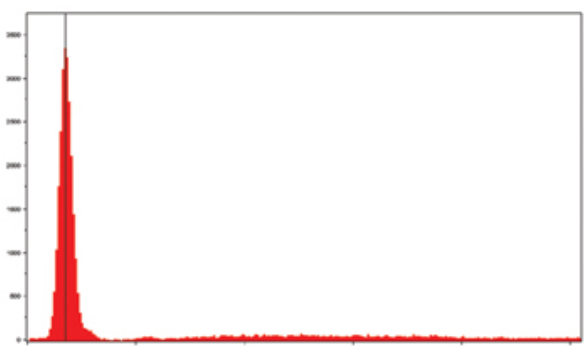

Boron

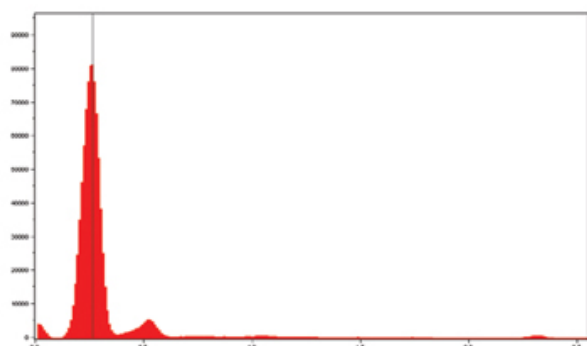

Carbon

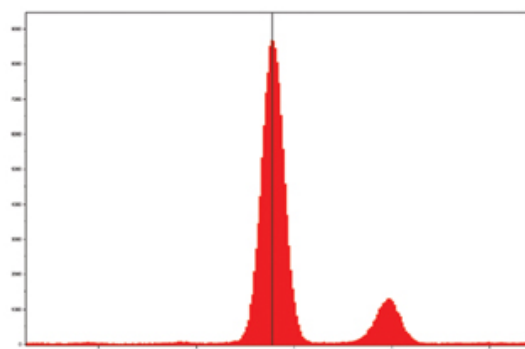

$129 \mathrm{eV}$ Mn sample spectrum 